\title{
Tan's contact of a harmonically trapped one-dimensional Bose gas: Strong-coupling expansion and conjectural approach at arbitrary interactions
}

\author{
Guillaume Lang ${ }^{1, a}$, Patrizia Vignolo ${ }^{2}$, and Anna Minguzzi ${ }^{1}$ \\ 1 Université Grenoble-Alpes, LPMMC, F-38000 Grenoble, France and CNRS, LPMMC, \\ 38000 Grenoble, France \\ 2 Université Côte d'Azur, CNRS, Institut Non Linéaire de Nice, 1361 route des Lucioles, \\ 06560 Valbonne, France
}

Received 25 October 2016 / Received in final form 27 December 2016 Published online 25 May 2017

\begin{abstract}
We study Tan's contact, i.e. the coefficient of the highmomentum tails of the momentum distribution at leading order, for an interacting one-dimensional Bose gas subjected to a harmonic confinement. Using a strong-coupling systematic expansion of the ground-state energy of the homogeneous system stemming from the Bethe-Ansatz solution, together with the local-density approximation, we obtain the strong-coupling expansion for Tan's contact of the harmonically trapped gas. Also, we use a very accurate conjecture for the ground-state energy of the homogeneous system to obtain an approximate expression for Tan's contact for arbitrary interaction strength, thus estimating the accuracy of the strong-coupling expansion.Our results are relevant for ongoing experiments with ultracold atomic gases.
\end{abstract}

\section{Introduction}

Continuous experimental progresses in trapping and cooling atomic gases have led to the realization of one-dimensional (1D) geometries, the observation of the effect of quantum fluctuations $[1,2]$, and the reach of the strongly correlated, Tonks-Girardeau regime $[3,4]$, where many of the properties of bosons are the same as those of free fermions [5]. Several physical properties have been experimentally studied, e.g. the density profiles [3], the two- and three-body correlation functions [6,7], the collective excitation spectrum [8,9], and transport of an impurity in a 1D gas [10] (see e.g. [11] for a comprehensive review).

One of the most common experimental observables is the momentum distribution. This quantity embeds the one-body properties of the gas, in particular it corresponds to the Fourier transform of the one-body density matrix, yielding information on the

\footnotetext{
a e-mail: guillaume.lang@lpmmc.cnrs.fr
} 
first-order spatial coherence of the gas. At zero temperature, an interacting 1D Bose gas is predicted to display quasi-off-diagonal long-range order, i.e. an algebraic decay of the one-body density matrix at large distances [12]. High-precision measurements of the momentum distribution are becoming available [13], calling for new theoretical developments.

Recent experimental studies allow to access in particular the high-momentum region of the momentum distribution $[14,15]$. The high-momentum tails of the momentum distribution $n(k)$ of a gas with contact interactions display a universal $n(k) \propto 1 / k^{4}$ decay $[16,17]$, which originates from the zero-range interaction potential: bosons with contact interactions have a cusp in the many-body wavefunction whenever the relative distance of each pair of particles vanishes. This implies a non-analyticity in the one-body density matrix at short distances, in the form $\rho_{1}(x) \sim|x|^{3}$, which leads to the high-momentum tails.

The weight of the momentum distribution tails, known as Tan's contact, is an important two-body quantity, related to various physical observables, such as the interaction energy, the two-body correlation function at zero distance, the rfspectroscopy, through Tan's relations [18-23]. For a homogeneous 1D Bose gas, Tan's contact at zero temperature can be obtained exactly using the Bethe Ansatz solution for the ground-state energy $[17,24,25]$. In the experiments, ultracold atomic gases are kept together by an external trap, which is in most cases a harmonic confinement. Tan's contact of an interacting one-dimensional Bose gas under harmonic confinement has been first studied in reference [17], where a numerical solution based on the local-density approximation (LDA) of the homogeneous-system result has been provided. Analytically, again in the local-density approximation, a strong-coupling expansion has been derived to zeroth order [17], as well as corrections to first and second order [26]. A comparison with matrix-product state simulations has shown that the local-density approximation works surprisingly well [26], even for a small number of particles.

The study of the equation of state of an interacting 1D Bose gas has received a renewed attention $[25,27]$. In particular, in reference [25] we have derived a strongcoupling expansion for the ground-state energy to an unprecedented accuracy as well as a conjectural expression which is extremely close to the exact numerical solution for a wide range of interaction strengths. Using the above results combined with the local-density approximation, in this work we propose a method to obtain the strongcoupling expansion for Tan's contact of a 1D Bose gas under harmonic confinement to arbitrary order. We also use the conjecture for the ground-state energy to obtain Tan's contact for all interaction regimes, from weak to strong coupling, as well as the lowest-order term in the weak-coupling expansion.

\section{Local-density approximation for Tan's contact of a 1D Bose gas}

We consider $N$ bosonic atoms of mass $m$ at zero temperature, confined by a tight atomic waveguide to a one-dimensional geometry. The atoms interact via a contact potential $v\left(x-x^{\prime}\right)=g \delta\left(x-x^{\prime}\right)$, where $g$ is the one-dimensional interaction constant, related to the three-dimensional scattering length of the atoms [28]. In the longitudinal direction the atoms are further confined by an external harmonic confinement $V_{\text {ext }}(x)=m \omega_{0}^{2} x^{2} / 2$, describing the optical or magnetic trapping present in the experiments with ultracold atoms. The Hamiltonian of the system reads

$$
H=\sum_{j=1}^{N}\left[\frac{-\hbar^{2}}{2 m} \frac{\partial^{2}}{\partial x_{j}^{2}}+V_{e x t}\left(x_{j}\right)+g \sum_{\ell>j} \delta\left(x_{j}-x_{\ell}\right)\right]
$$


In the homogeneous system, the interaction strength is measured in terms of the dimensionless parameter $\gamma=m g /\left(\hbar^{2} \rho\right)$, where $\rho$ is the average linear density of the homogeneous gas. In the case of harmonic confinement, introducing the harmonicoscillator length $a_{h o}=\sqrt{\hbar /\left(m \omega_{0}\right)}$ and the one-dimensional scattering length $a_{1 \mathrm{D}}=$ $-2 \hbar^{2} /(\mathrm{gm})$, the corresponding dimensionless parameter is $\alpha_{0}=2 a_{h o} /\left(\left|a_{1 \mathrm{D}}\right| \sqrt{N}\right)[29]$.

According to Tan's sweep relation, Tan's contact in 1D is related to the groundstate energy $E$ of the gas according to [30]

$$
C=-\frac{m^{2}}{\pi \hbar^{4}} \frac{\partial E}{\partial(1 / g)} .
$$

In order to determine Tan's contact of the harmonically-confined gas, we employ the density-functional approach developed in [26]. In detail, we define a functional $E[\rho]$ of the density $\rho(x)$ which, in the local-density approximation, reads

$$
E[\rho]=\int d x\left[\epsilon(\rho)+\left(V_{e x t}(x)-\mu\right) \rho(x)\right],
$$

where $\epsilon$ is the ground-state energy density of the homogeneous gas. The ground-state density profile is obtained by minimizing the energy functional, i.e. setting $\delta E / \delta \rho=0$. This yields an implicit equation for the density profile,

$$
\frac{3}{2} \frac{\hbar^{2}}{m} \rho^{2} e(\gamma)-\frac{g \rho}{2} \frac{\partial e}{\partial \gamma}=\mu-V_{e x t}(x)=\mu\left(1-\frac{x^{2}}{R_{T F}^{2}}\right),
$$

where $R_{T F}=\sqrt{2 \mu /\left(m \omega_{0}^{2}\right)}$ is the Thomas-Fermi radius in a harmonic trap, and the dimensionless average ground-state energy per particle $e$ is such that $\epsilon(\rho)=\frac{\hbar^{2}}{2 m} \rho^{3} e(\gamma)$. The chemical potential $\mu$ is fixed by imposing the normalization condition $N=$ $\int d x \rho(x)$.

Combining equations (2) and (3), together with the inhomogeneous density profile (4), we obtain Tan's contact within the LDA:

$$
C_{L D A}=\left.g^{2} \frac{m^{2}}{2 \pi \hbar^{4}} \int d x \rho^{2}(x) \frac{\partial e}{\partial \gamma}\right|_{\rho=\rho(x)} .
$$

This expression readily generalizes the known result for the homogeneous system (see e.g. Ref. [31]):

$$
C=g^{2} \frac{m^{2}}{2 \pi \hbar^{4}} L \rho^{2} \frac{\partial e}{\partial \gamma} .
$$

In practice, since $e(\gamma)$ is not known analytically for the Lieb-Liniger model, one needs to rely on an approximation scheme to obtain Tan's contact in the homogeneous case, and in the trap within the LDA. We proceed by detailing procedures based on various approximations that allow, in principle, to reach excellent accuracy over the whole interaction range.

\section{Scaling relations and methods}

In this section, we combine the various relations obtained above to systematically compute Tan's contact with increasing accuracy. For the energy density $\epsilon(\rho)$, we take 
the solution stemming from the Lieb-Liniger Bethe Ansatz solution, and use various schemes to estimate $e(\gamma)$, i.e. a strong-coupling expansion

$$
e(\gamma)=\sum_{k=0}^{+\infty} \frac{a_{k}}{\gamma^{k}}
$$

where $\left\{a_{k}\right\}_{k \geq 0}=\left\{\pi^{2} / 3,-4 \pi^{2} / 3,4 \pi^{2}, \ldots\right\}$ is currently known up to order 20 [25], as well as the conjecture

$$
e(\gamma)=\frac{\gamma^{2}}{3} \sum_{k=0}^{+\infty} \frac{\pi^{2 k+2} P_{k}(\gamma)}{(2+\gamma)^{3 k+2}}
$$

where $\left\{P_{k}(X)\right\}_{k>0}=\left\{1, \frac{32}{15},-\frac{96}{35} X+\frac{848}{315}, \ldots\right\}$ are polynomials explicitly known up to order 6 [25]. Expression (8) is very close to the exact numerical Bethe Ansatz solution for a wide range of interaction strengths. It is also a priori possible to rely on a conjectural expansion in the weakly-interacting regime,

$$
e(\gamma)=\sum_{k=0}^{+\infty} a_{k}^{\prime} \gamma^{1+k / 2}
$$

where $\left\{a_{k}^{\prime}\right\}_{k \geq 0}=\left\{1,-\frac{4}{3 \pi}, \frac{1}{6}-\frac{1}{\pi^{2}}, \ldots\right\}$ is analytically known up to second order at the time being [32]. Higher-order terms were obtained numerically in a very recent work and the exact value of a few of them has been guessed [33].

\subsection{Strong-coupling expansion for Tan's contact}

We first derive the strong-coupling expansion of Tan's contact for a harmonically trapped gas, based on the strong-coupling expansion equation (7) for the ground state-energy of the homogeneous system. Combining equation (4), the normalization condition, and the relation $g=\hbar \omega_{0} a_{h o} \sqrt{N} \alpha_{0}$, the natural rescaled variables are $\bar{\rho} \equiv$ $\rho a_{h o} / \sqrt{N}, \bar{\mu} \equiv \mu /\left(N \hbar \omega_{0}\right)$ and $\bar{x} \equiv x / R_{T F}$, whereupon we obtain the following set of equations:

$$
\frac{1}{2} \sum_{k=0}^{+\infty} \frac{A_{k}}{\alpha_{0}^{k}} \bar{\rho}^{k+2}\left(\bar{x}, \alpha_{0}\right)=\left(1-\bar{x}^{2}\right) \bar{\mu}\left(\alpha_{0}\right),
$$

where $A_{k} \equiv(k+3) a_{k}$, and also

$$
1=\sqrt{2 \bar{\mu}} \int_{-1}^{1} d \bar{x} \bar{\rho}(\bar{x})
$$

A straightforward approach to solve the above equations proceeds as follows. One truncates the series in equation (10) to order $n$. Using the values for the coefficients $\left\{a_{k}\right\}_{k \leq n}$ from equation (7), one can express $\bar{\rho}$ as the root of a $(n+2)$ th-degree polynomial as a function of $\bar{\mu}$ and inject into equation (11) to find $\bar{\mu}$, and thus $\bar{\rho}$ explicitly, consistently to order $n$ by expanding the obtained solution in $1 / \alpha_{0}$. This approach, when carried analytically, suffers from two major drawbacks. First, it is limited to $n=2$ since in general, polynomials of order strictly higher than 4 can not be solved by radicals. Second, among the $n$ possible solutions, one has to select the physical one. Using Cardan's method for $n=1$ and Ferrari's method for $n=2$, putting the roots of the polynomials in a trigonometric form for simplicity, we have used this 
approach to compute the strong-coupling expansion of Tan's contact to second order in $1 / \alpha_{0}$. This has also been useful to benchmark the more general method described just below.

In order to obtain higher-order terms in the strong-coupling expansion, we have developed a more efficient procedure, which allows to systematically compute the expansion of Tan's contact to arbitrary order. The latter relies on the following expansions: $\bar{\mu}=\sum_{k=0}^{+\infty} \frac{c_{k}}{\alpha_{0}^{k}}$, and $\bar{\rho}(\bar{x})=\sum_{j=0}^{+\infty} \frac{b_{j}}{\alpha_{0}^{j}} f_{j}(\bar{x})$, where $\left\{f_{j}\right\}_{j \geq 0}$ is a set of unknown functions, injected into equations $(10,11)$. By analysis, we find that the consistency condition at a given order converts into $b_{j} f_{j}(\bar{x})=\sum_{m=0}^{j} b_{m j}\left(1-\bar{x}^{2}\right)^{(m+1) / 2}$, where $\left\{b_{m j}\right\}$ are unkwown coefficients of an upper triangular matrix. Then, synthesis yields:

$$
\frac{1}{2} \sum_{k=0}^{+\infty} \frac{A_{k}}{\alpha_{0}^{k}}\left(\sum_{j=0}^{+\infty} \frac{1}{\alpha_{0}^{j}} \sum_{m=0}^{j} b_{m j}\left(1-\bar{x}^{2}\right)^{(m+1) / 2}\right)^{k+2}=\left(1-\bar{x}^{2}\right) \sum_{k=0}^{+\infty} \frac{c_{k}}{\alpha_{0}^{k}}
$$

and

$$
1=32 \sum_{k=0}^{+\infty} \frac{c_{k}}{\alpha_{0}^{k}}\left[\sum_{j=0}^{+\infty} \frac{1}{\alpha_{0}^{j}} \sum_{m=0}^{j} b_{m j} 2^{m} B\left(\frac{m+3}{2}, \frac{m+3}{2}\right)\right]^{2}
$$

where $B$ is the Euler Beta function. Equations (12) and (13) are the final set of equations. As can be seen, solving the system truncated to order $n$ requires the solution at all lower orders, thus the procedure becomes increasingly lengthy. Moreover, at each step equation (12) splits into $n+1$ independent equations, obtained by equating the coefficients of $\left(1-\bar{x}^{2}\right)_{m=0, \ldots, n}^{(1+m) / 2}$ in the LHS and RHS. One thus needs to solve a system of $n+2$ equations to obtain $c_{n}$ and $\left\{b_{m n}\right\}_{m=0, \ldots, n}$, but fortunately, $n$ of them, giving $b_{m j}, m \geq 1$, are fully decoupled.

As a final step, using equation (5), we obtain Tan's contact. In natural units imposed by the scaling, i.e. taking $\bar{C}_{L D A}=C_{L D A} a_{h o}^{3} / N^{5 / 2}$, we have

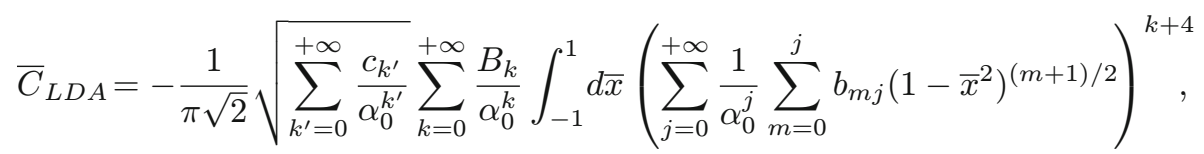

where $B_{k}=(k+1) a_{k+1}$. Note that the value is positive because $a_{1}<0$. At order $n$, the condition $k^{\prime}+k+j^{\prime}=n$, where $j^{\prime}$ is the power of $\alpha_{0}$ in the integrand, shows that the coefficient of order $n$ is a sum of $\left(\begin{array}{c}n+2 \\ n\end{array}\right)$ integrals. One of them involves $a_{n+1}$, so one needs to know the function $e(\gamma)$ to order $n+1$ in $1 / \gamma$ to obtain the expansion of the Tan's contact to order $n$ in $1 / \alpha_{0}$.

\subsection{Tan's contact at arbitrary interactions from the conjecture}

To check the validity of the strong-coupling method, we have derived another resolution scheme, from equation (8). After rescaling and straightforward algebra, one obtains the mixed form

$$
\frac{\alpha_{0}^{2}}{6} \sum_{k=0}^{+\infty} \frac{\pi^{2 k+2}}{(\gamma+2)^{3 k+3}}\left[(\gamma+2) P_{k}(\gamma)-\gamma(\gamma+2) P_{k}^{\prime}(\gamma)+3 k+2\right]=\bar{\mu}\left(\alpha_{0}\right)\left(1-\bar{x}^{2}\right)
$$


Once truncated at order $n$, it yields a polynomial in $1 /(\gamma+2)$ whose roots are found numerically. We proceed as follows. We start from a guessing value of $\bar{\mu}$ and from equation $(15)$ we obtain $\bar{\rho}(\bar{\mu}, \bar{x})$. We integrate the density and we use the difference $N-\int \rho d x$ as control parameter of the accuracy of the initial value for $\bar{\mu}$. A findroot subroutine exploits this contol parameter to converge to the $\bar{\mu}$ value that ensures the density normalization to $N$ bosons. Once the correct density is computed, we get $C_{L D A}$ from equation (5).

\subsection{Weak-coupling expansion for Tan's contact}

At weak iteractions, we derive an expression for Tan's contact using the weak-coupling expansion equation (9). Using the same notations as above, we obtain

$$
\sum_{k=0}^{+\infty} \frac{a_{k}^{\prime}}{4}(4-k) \bar{\rho}^{\frac{2-k}{2}}\left(\bar{x}, \alpha_{0}\right) \alpha_{0}^{\frac{k+2}{2}}=\left(1-\bar{x}^{2}\right) \bar{\mu}\left(\alpha_{0}\right) .
$$

On the other hand, the normalization condition is given by equation (11) as before. Here, it is not obvious to what order one should truncate the expressions to obtain a consistent expansion at given order, nor to find the variable in which to expand, as can be seen by evaluating the first orders. Considering only the $k=0$ term in the sum, one easily finds $\bar{\rho}(\bar{x})=(9 / 32)^{1 / 3}\left(1-\bar{x}^{2}\right) / \alpha_{0}^{1 / 3}$ and $\bar{\mu}\left(\alpha_{0}\right)=(9 / 32)^{1 / 3} \alpha_{0}^{2 / 3}$. The expansion to next order is problematic. If one retains terms up to $k=1$, corresponding to the Bogoliubov approximation, since the coefficient $a_{1}^{\prime}$ is negative, the equation of state at sufficiently large density becomes negative [24]. Then it is not possible to use it to perform the local-density approximation. One may also recall that the localdensity approximation breaks down at very weak interactions, where it is not accurate to neglect the quantum tails in the density profile. In this regime, a different scaling parameter becomes relevant $[34,35]$.

\section{Results for Tan's contact}

Following the approach presented in Section 3.1, the strong-coupling expansion reads:

$$
\begin{aligned}
\bar{C}_{L D A}= & \frac{128 \sqrt{2}}{45 \pi^{3}}+\frac{1}{\alpha_{0}}\left(-\frac{8192}{81 \pi^{5}}+\frac{70}{9 \pi^{3}}\right)+\frac{\sqrt{2}}{\alpha_{0}^{2}}\left(\frac{131072}{81 \pi^{7}}-\frac{30656}{189 \pi^{5}}-\frac{4096}{525 \pi^{3}}\right) \\
& +\frac{1}{\alpha_{0}^{3}}\left(-\frac{335544320}{6561 \pi^{9}}+\frac{4407296}{729 \pi^{7}}+\frac{872701}{2025 \pi^{5}}-\frac{112}{3 \pi^{3}}\right) \\
& +\frac{\sqrt{2}}{\alpha_{0}^{4}}\left(\frac{47982837760}{59049 \pi^{11}}-\frac{717291520}{6561 \pi^{9}}-\frac{108494512}{10935 \pi^{7}}+\frac{2112512}{1701 \pi^{5}}+\frac{65536}{2205 \pi^{3}}\right) .
\end{aligned}
$$

This expression agrees with the zero order one obtained in [17] and with the one derived for a $\kappa$-component balanced spinful Fermi gas in [26] to order 2: in fact one may find the bosonic result by taking the limit of very large number of fermionic components $\kappa \rightarrow \infty$ [36]. 


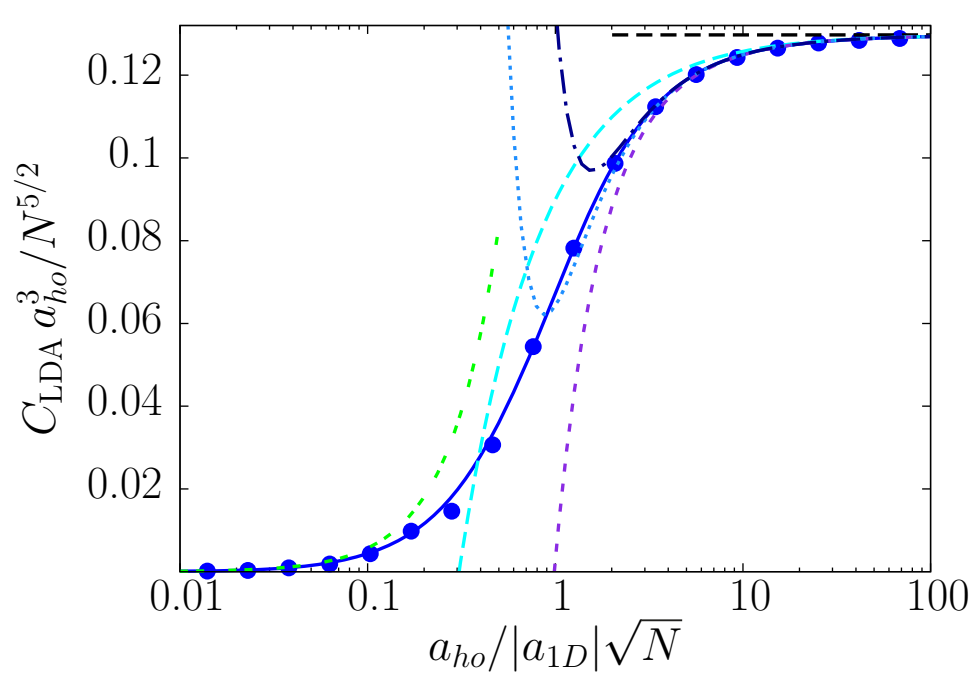

Fig. 1. Scaled Tan's contact for a 1D Bose gas (in units of $N^{5 / 2} / a_{h o}^{3}$ ) as a function of the dimensionless interaction strength $\alpha_{0} / 2=a_{h o} /\left(\left|a_{1 D}\right| \sqrt{N}\right)$. Results from the strong-coupling expansion (17): Tonks-Girardeau (horizontal long-dashed line, black), 1st order correction (long dashed, cyan), 2nd order correction (short-dashed, purple), 3rd order correction (dotted, light blue), 4th order correction (dot-dashed, dark blue). Results at arbitrary interactions: conjecture (8) to order six (blue dots), exact equation of state (data from Ref. [17], continuous, blue). We also show the weak-coupling expansion (18) (double dashed, green).

The weak-coupling expansion obtained from Section 3.3 reads:

$$
\bar{C}_{L D A}=\frac{1}{5 \pi}\left(\frac{3}{2}\right)^{2 / 3} \alpha_{0}^{5 / 3}
$$

in agreement with [17].

Figure 1 summarizes our results for Tan's contact. Notice that, although the contact is scaled by the overall factor $N^{5 / 2} / a_{h o}^{3}$, it still depends on the number of particles through the factor $\alpha_{0} / 2=a_{h_{o}} /\left|a_{1 D}\right| \sqrt{N}$. First, we notice that the results based on the conjecture are extremely close to the ones obtained from the full solution of the Bethe-Ansatz equation of state in reference [17]. Second, by comparing the strongcoupling expansion with the results of the full calculation, we notice that the expansion (17) is valid down to $\alpha_{0} / 2 \simeq 3$, and provides an useful analytical expression for Tan's contact in harmonic trap. In order to accurately describe the regime of lower interactions one would need a considerable number of terms in the strong-coupling expansion. The same is true for the series expansion for the ground-state energy of the homogenenous gas [25]. The use of the conjecture (8) is then a valuable alternative with respect to solving numerically the Bethe-Ansatz integral equations, the weak coupling expansion being applicable only for very weak interactions $\alpha_{0} / 2 \simeq 0.05$.

\section{Conclusions and outlook}

In conclusion, in this work we have determined Tan's contact for a harmonicallytrapped 1D Bose gas. In particular, using an asymptotic expansion of the groundstate energy of the homogeneous system and the local-density approximation, we 
have developed a general method to obtain a strong-coupling expansion of Tan's contact to arbitrary order, and have provided the coefficients for the expansion up to order four. We have tested it against a calculation of Tan's contact based on a conjecture of the ground-state energy at arbitrary interactions [25] as well as to the full solution of the Bethe-Ansatz equations provided in reference [17]. The strongcoupling expansion yields an accurate expression at large interactions, but requires a considerable number of terms to obtain good accuracy at intermediate interactions. In this parameter regime it is then useful to apply the method based on the conjecture for the equation of state. In outlook, the method presented in this work could be used to calculate higher-order terms of the strong coupling expansion with the aim of resumming the terms as was done to obtain the conjectural expression for the homogeneous gas. The local-density approximation could be tested by comparing with ab-initio numerical simulations. It would be interesting to generalize this method to the case of multicomponent $1 \mathrm{D}$ gases as well as to finite temperature, beyond the infinitely repulsive Tonks-Girardeau limit of references [31,37].

We thank M. Albert, M. Rizzi and J. Decamp for discussions. We thank V. Dunjko and M. Olshanii for providing us the data of reference [17]. We acknowledge financial support from the ANR projects Mathostaq (ANR-13-JS01-0005-01) and SuperRing (ANR-15-CE300012-02). AM wishes to dedicate this work to Roger Maynard, which follows his continuous encouragement and enthusiasm.

\section{References}

1. S. Dettmer, D. Hellweg, P. Ryytty, J.J. Arlt, W. Ertmer, K. Sengstock, D.S. Petrov, G.V. Shlyapnikov, H. Kreutzmann, L. Santos, M. Lewenstein, Phys. Rev. Lett. 87, $160406(2001)$

2. J. Esteve, J.-B. Trebbia, T. Schumm, A. Aspect, C.I. Westbrook, I. Bouchoule, Phys. Rev. Lett. 96, 130403 (2006)

3. T. Kinoshita, T. Wenger, D.S. Weiss, Science 305, 1125 (2004)

4. B. Paredes, A. Widera, V. Murg, O. Mandel, S. Fölling, I. Cirac, G.V. Shlyapnikov, T.W. Hänsch, I. Bloch, Nature 429, 277 (2004)

5. M. Girardeau, J. Math. Phys. 1, 516 (1960)

6. T. Kinoshita, T. Wenger, D.S. Weiss, Phys. Rev. Lett. 95, 190406 (2005)

7. E. Haller, M. Rabie, M.J. Mark, J.G. Danzl, R. Hart, K. Lauber, G. Pupillo, H.-C. Nägerl, Phys. Rev. Lett. 107, 230404 (2011)

8. E. Haller, M. Gustavsson, M.J. Mark, J.G. Danzl, R. Hart, G. Pupillo, H.-C. Nägerl, Science 325, 5945 (2009)

9. B. Fang, G. Carleo, A. Johnson, I. Bouchoule, Phys. Rev. Lett. 113, 035301 (2014)

10. S. Palzer, C. Zipkes, C. Sias, M. Köhl, Phys. Rev. Lett. 103, 150601 (2009)

11. M.A. Cazalilla, R. Citro, T. Giamarchi, E. Orignac, M. Rigol, Rev. Mod. Phys. 83, 1405 (2011)

12. F.D.M. Haldane, Phys. Rev. Lett. 47, 1840 (1981)

13. T. Jacqmin, B. Fang, T. Berrada, T. Roscilde, I. Bouchoule, Phys. Rev. A 86, 043626 (2012)

14. R. Chang, Q. Bouton, H. Cayla, C. Qu, A. Aspect, C.I. Westbrook, D. Clément, arXiv:1608.04693

15. R.J. Fletcher, R. Lopes, J. Man, N. Navon, R.P. Smith, M.W. Zwierlein, Z. Hadzibabic, arXiv: 1608.04377

16. A. Minguzzi, P. Vignolo, M. Tosi, Phys. Lett. A 294, 222 (2002)

17. M. Olshanii, V. Dunjko, Phys. Rev. Lett. 91, 090401 (2003)

18. S. Tan, Ann. Phys. 323, 2952 (2008)

19. S. Tan, Ann. Phys. 323, 2971 (2008) 
20. S. Tan, Ann. Phys. 323, 2987 (2008)

21. E. Braaten, D. Kang, L. Platter, Phys. Rev. A 78, 053606 (2008)

22. E. Braaten, L. Platter, Phys. Rev. Lett. 100, 205301 (2008)

23. E. Braaten, D. Kang, L. Platter, Phys. Rev. Lett. 106, 153005 (2011)

24. E.H. Lieb, W. Liniger, Phys. Rev. 130, 1605 (1963)

25. G. Lang, F. Hekking, A. Minguzzi (2016), arXiv:1609.08865

26. J. Decamp, J. Jünemann, M. Albert, M. Rizzi, A. Minguzzi, P. Vignolo, Phys. Rev. A 94, $053614(2016)$

27. Z. Ristivojevic, Phys. Rev. Lett. 113, 015301 (2014)

28. M. Olshanii, Phys. Rev. Lett. 81, 938 (1998)

29. C. Menotti, S. Stringari, Phys. Rev. A 66, (043610) 2002

30. M. Barth, W. Zwerger, Ann. Phys. 326, 2544 (2011)

31. W. Xu, M. Rigol, Phys. Rev. A 92, 063623 (2015)

32. C.A. Tracy, H. Widom, J. Phys. A 49, 294001 (2016)

33. S. Prolhac (2016), arXiv:1610.08912v3

34. A. Iu. Gudyma, G.E. Astrakharchikm, M. Zvonarev, Phys. Rev. A 92, 021601 (2014)

35. S. Choi, V. Dunjko, Z.D. Zhang, M. Olshanii, Phys. Rev. Lett. 115, 115302 (2015)

36. C.N. Yang, Y. Yi-Zhuang, Chin. Phys. Lett. 28, 020503 (2011)

37. P. Vignolo, A. Minguzzi, Phys. Rev. Lett. 110, 020403 (2013)

Open Access This is an Open Access article distributed under the terms of the Creative Commons Attribution License (http://creativecommons.org/licenses/by/4.0), which permits unrestricted use, distribution, and reproduction in any medium, provided the original work is properly cited. 